\title{
Identification modale déterministe par la méthode des sous-espaces avec prévision de l'intervalle d'erreur sur les paramètres modaux
}

\author{
Michel Raffy et Camille Gontier ${ }^{\mathrm{a}}$ \\ Laboratoire de Mécanique et de Rhéologie, École Nationale d'Ingénieurs du Val de Loire, rue de la chocolaterie, 41000 Blois, \\ France
}

Reçu le 29 juillet 2004, accepté le 15 avril 2006

\begin{abstract}
Résumé - Le présent article développe une approche permettant d'évaluer les erreurs statistiques commises sur les paramètres modaux obtenus lors d'une identification déterministe, c'est-à-dire lorsque l'excitation peut être mesurée. À partir des résultats d'une identification, la question de la validité des résultats se pose, en particulier en ce qui concerne les taux d'amortissement, souvent identifiés avec une faible précision. L'évaluation des erreurs sur ces paramètres permet alors de fournir un intervalle de confiance, et par là d'évaluer la qualité de l'identification. À partir de l'algorithme d'identification de sous-espaces N4SID, l'article développe une formulation permettant d'obtenir les erreurs statistiques sur les paramètres obtenus. Cette formulation est ensuite validée et discutée sur l'exemple d'un système masse-ressort-amortisseur à 5 degrés de liberté, puis sur une structure expérimentale représentant un bâtiment à deux étages.
\end{abstract}

Mots clés : Analyse modale / identification / méthodes sous-espaces / statistiques / intervalle de confiance

\begin{abstract}
Deterministic modal identification from a subspace method with estimation of the confidence interval on modal parameters. The present paper develops a theory providing the statistical errors on the modal parameters obtained from an identification procedure in testing conditions, i.e. when the excitation can be measured. Once modal parameters are determined, the question of data reliability arises especially concerning damping ratios, which are often identified with an unsatisfactory accuracy. For these reasons, providing a confidence range with any estimated parameter would be the only way to ensure valuable information. From a deterministic subspace identification algorithm such as N4SID, the present paper develops a formulation which allows the evaluation of the statistical error on the identified parameters. After presenting the basic formula, the method is validated and discussed by means of, first an example of spring-damper-mass system with 5 degrees of freedom, second an experimental structure representing a two-floor building.
\end{abstract}

Key words: Modal analysis / identification / subspace methods / statistics / confidence intervals

\section{Introduction}

Parmi les nombreuses méthodes disponibles aujourd'hui dans le domaine de l'analyse modale, les méthodes dites «temporelles » [1] sont de plus en plus employées, comme alternatives aux méthodes « fréquentielles » classiques [2]. Parmi celles-ci, les méthodes dites « de sousespaces » sont spécialement attractives car ne demandant pas de manipulations préalables des données. Une vue d'ensemble de ces méthodes peut être trouvée dans

a Auteur correspondant :

camille.gontier@univ-tours.fr la littérature $[3,4]$. Ces méthodes sont couramment employées dans trois situations typiques : i) identification déterministe, ii) identification purement stochastique, iii) identification combinée déterministe-stochastique. La méthode proposée dans le présent article est développée dans le cadre de la troisième situation, mais peut être facilement transposée pour répondre à la deuxième configuration.

Dans ces situations, la procédure d'identification est de nature stochastique. Ainsi la question se pose de l'erreur commise sur les paramètres modaux identifiés, et cela quel que soit le type d'algorithme utilisé. En général, les fréquences modales sont identifiées avec une assez bonne 


\section{Nomenclature}

$M, \zeta, K$ matrices dynamiques d'une structure

$u, z$ excitation et réponse de la structure

$A_{\mathrm{c}}, B_{\mathrm{c}}, C_{\mathrm{c}}, D_{\mathrm{c}}$, matrices dynamiques de l'équation

d'état continue

$A, B, C, D$ matrices dynamiques de l'équation

d'état discrète

$l, m, n$ nombre de mesures, d'excitateurs,

de degrés de liberté

$x, y$ vecteurs d'état et de mesure

$w, v$ bruits de process et de mesure

$\beta, r$ ordre du modèle et rang de troncature

$\lambda_{k}, \mu_{k}$ valeurs propres des matrices $A_{\mathrm{c}}$ et $A$

$\omega_{k}, \eta_{k}$ pulsations propres et taux d'amortissement

$\Gamma$ matrice d'observabilité étendue

$U, S, V$ matrices de la décomposition en valeurs singulières

$(\bullet)_{k} \bullet,(\bullet) \bullet k_{k} k^{\text {ième }}$ ligne et $k^{\text {ième }}$ colonne de la matrice $\bullet$

$\delta T$ période d'échantillonnage

• erreur sur la variable

- estimée de la variable •

- transposée conjuguée complexe de la variable •

$\bullet^{\mathrm{T}}, \bullet^{\mathrm{C}}$ transposée et conjuguée de la variable •

- ${ }^{\dagger}$ pseudo inverse de la matrice •

$\prod_{B}$ opérateur de projection orthogonale

$\prod_{B \perp}$ opérateur de projection orthogonale complémentaire

$/{ }_{B} C$ opérateur de projection oblique

Procédures génériques $(\mathrm{N})$ : un vecteur signal $s(t)$,

un indice de temps $k$, tel que $t=k \delta T$ et une taille $i$ étant donnés, un vecteur signal étendu $s_{k}$ aussi noté $s_{k, i}$ est défini :

$s_{k} \equiv s_{k, i}=\left[s(k)^{\mathrm{T}} \quad s(k+1)^{T} \ldots s(k+i-1)^{T}\right]^{T}$

À partir de celui-ci, une longueur de séquence $j$ étant donnée, une «matrice signal » de Hankel $S$, notée

aussi $S_{k, i, j}$, est établie comme suit :

$S_{k} \equiv S_{k, i, j}=\left[\begin{array}{ll}s_{k} & s_{k+1} \ldots s_{k+j-1}\end{array}\right]$

Deux indices $\alpha$ et $\beta$ étant fixés par l'utilisateur, une matrice dite du «passé $» S_{\mathrm{p}}$ et une matrice dite du « futur $S_{\mathrm{f}}$ sont définies à partir des procédures (N1) et (N2) comme suit :

$S_{\mathrm{p}}=S_{k, \alpha, j}=\left[\begin{array}{ll}s_{k, \alpha} & s_{k+1, \alpha} \ldots s_{k+j-1, \alpha}\end{array}\right]$

$S_{\mathrm{f}}=S_{k+\alpha, \beta, j}=\left[\begin{array}{ll}s_{k+\alpha, \beta} & s_{k+\alpha+1, \beta} \ldots s_{k+\alpha+j-1, \beta}\end{array}\right]$

En fin de compte, les matrices $S_{\mathrm{p}}$ et $S_{\mathrm{f}}$ se présentent comme suit :

$S_{\mathrm{p}}=\left[\begin{array}{cccc}s(k) & s(k+1) & \ldots & s(k+j-1) \\ s(k+1) & s(k+2) & \ldots & s(k+j) \\ \ldots & \ldots & \ldots & \\ s(k+\alpha-1) & s(k+\alpha) & \ldots & s(k+\alpha+j-1)\end{array}\right]$
$S_{\mathrm{f}}=\left[\begin{array}{cccc}s(k+\alpha) & s(k+\alpha+1) & \ldots & s(k+\alpha+j-1) \\ s(k+\alpha+1) & s(k+\alpha+2) & \ldots & s(k+\alpha+j) \\ \ldots & \ldots & \ldots & \ldots \\ s(k+2 \alpha-1) & s(k+2 \alpha) & \ldots & s(k+2 \alpha+j-1)\end{array}\right]$

Procédures spécifiques $(\mathrm{P})$ : un signal vectoriel $x(t)$,

une longueur de séquence $j$ et un indice $k$ étant donnés,

une séquence étendue de vecteurs est définie comme suit :

$X_{k}=[x(k) \quad x(k+1) \ldots x(k+j-1)]$

À partir de cette procédure deux séquences de vecteurs

dites du «passé » $X_{\mathrm{p}}$ et du « futur » $X_{\mathrm{f}}$ sont définies

comme suit : $X_{\mathrm{p}}=x_{k, j}$

$$
X_{\mathrm{f}}=x_{k+\beta, j}
$$

précision, ce qui n'est malheureusement pas le cas pour les taux d'amortissement. Pour ces raisons, il serait judicieux de pouvoir fournir, associé à chaque paramètre modal identifié, un intervalle d'erreur permettant d'assurer la validité des informations fournies.

Le problème de l'étude statistique des paramètres identifiés, à partir des méthodes de sous-espaces, a été abordée par plusieurs auteurs au cours de la dernière décennie. Parmi ceux-ci, on pourra citer Viberg [4-6], Jansson [7], Bauer [8-10], Deistler [11], Peternell [12] et Knudsen [13], qui ont principalement étudié les conditions de consistance des méthodes de sous-espaces. De ce fait, ils ont fourni dans le même temps des éléments de base permettant d'envisager l'estimation d'une erreur statistique associée aux paramètres modaux identifiés.

Dans le présent article, une méthode proposée par Viberg [6], réécrite dans le cadre de l'algorithme N4SID [3], formulée en termes d'accélérations [14], et de l'algorithme IVM [4], est adaptée pour fournir l'erreur statistique associée à chacun des paramètres modaux identifiés. La présente théorie sera alors appliquée, au titre de validation, sur des données provenant d'un système simulé masse-ressort-amortissseur.

La littérature concernant l'erreur sur les paramètres modaux présente peu d'illustrations concernant des structures réelles, et aucune, à notre connaissance, conduisant à validation de la méthode dans le cadre des approches de sous-espaces. Au-delà d'une légère reformulation théorique, en particulier pour incorporer le contexte « déterministe » où les excitations sont connues, l'ambition de cet article est précisément de confronter la théorie à une expérimentation réelle, étape indispensable mais trop souvent occultée vers l'application industrielle des méthodes.

\section{2 Équations dynamiques et hypothèses}

L'équation dynamique d'une structure à $n$ degrés de liberté, sous les hypothèses de linéarité et d'invariance dans le temps des paramètres, est usuellement exprimée sous la forme

$$
M \ddot{z}(t)+\zeta \dot{z}(t)+K z(t)=B_{a} u(t)
$$

où les matrices $M, \zeta, K$ de dimensions $n \times n$ représentent respectivement les matrices de masse, d'amortissement et de raideur de la structure. Les vecteurs $z$, $\dot{z}$ et $\ddot{z}$, de dimensions $n$, définissent les déplacements, les vitesses et les accélérations des $n$ degrés de liberté de la structure. Les symboles $B_{a}$ et $u$ désignent respectivement la matrice d'excitation (dimensions $n \times m$ ), et le vecteur des forces extérieures (dimension $m$ ).

On définit usuellement le vecteur des variables d'état

$$
x(t)=\left[z(t)^{T} \dot{z}(t)^{T}\right]^{T}
$$

L'équation (2.1) prend alors la forme

$$
\dot{x}(t)=A_{\mathrm{c}} x(t)+B_{\mathrm{c}} u(t)
$$


à condition de poser

$$
A_{\mathrm{c}}=\left[\begin{array}{cc}
0 & I \\
-M^{-1} K & -M^{-1} \zeta
\end{array}\right] \text { et } B_{\mathrm{c}}=\left[\begin{array}{c}
0 \\
M^{-1} B_{a}
\end{array}\right]
$$

Généralement, seul un petit nombre de degrés de liberté de la structure est observé. On définit un vecteur « d'observation $» y(t)$, de sorte que le système d'équations d'état en temps continu s'écrit :

$$
\left\{\begin{array}{l}
\dot{x}(t)=A_{\mathrm{c}} x(t)+B_{\mathrm{c}} u(t) \\
y(t)=C_{\mathrm{c}} x(t)+D_{\mathrm{c}} u(t)
\end{array}\right.
$$

Les matrices $A_{\mathrm{c}}$ et $B_{\mathrm{c}}$ (dimensions $2 n \times 2 n$ et $\left.2 n \times m\right)$, sont respectivement les matrices d'évolution et d'excitation du système, $C_{\mathrm{c}}$ (dimensions $\left.l \times 2 n\right)$ la matrice d'observation, et $D_{\mathrm{c}}$ (dimensions $\left.l \times m\right)$ la matrice de transmission directe des entrées vers les sorties.

Lors de l'acquisition des données, les signaux sont échantillonnées à une période $\delta T$. Les équations d'état en temps continu doivent être discrétisées en utilisant une procédure classique. Les équations d'état en temps discret qui en résultent s'écrivent :

$$
\left\{\begin{array}{l}
x(k+1)=A_{\mathrm{d}} x(k)+B_{\mathrm{d}} u(k)+w(k) \\
y(k)=C_{\mathrm{d}} x(k)+D_{\mathrm{d}} u(k)+v(k)
\end{array}\right.
$$

où les vecteurs $\mathrm{w}(\mathrm{k})$ et $\mathrm{v}(\mathrm{k})$, de dimensions respectives $2 n$ et $l$, représentent les bruits de process et de mesure.

La réponse de la structure étant généralement observée à l'aide d'accéléromètres, la formulation doit être réécrite en termes d'accélérations. On utilisera la forme suivante [14] :

$$
\left\{\begin{array}{l}
\dot{x}(k+1)=A \dot{x}(k)+B \delta u(k)+\dot{w}(k) \\
\ddot{y}(k)=C \dot{x}(k)+D \delta u(k)+\ddot{v}(k)
\end{array}\right.
$$

avec $A=A_{\mathrm{d}}=e^{\delta T A_{\mathrm{c}}}, B=A B_{\mathrm{c}}, C=C_{\mathrm{d}}=C_{\mathrm{c}}, D=$ $D_{\mathrm{d}}=D_{\mathrm{c}}$ et $\delta u(k)=u(k+1)-u(k)$

En ce qui concerne l'excitation du système, on posera les hypothèses suivantes :

a) Les bruits de process et de mesure sont des bruits blancs gaussiens de moyennes nulles, satisfaisant aux conditions de stationnarité et d'ergodicité, et de covariances

$$
E\left[\left[\begin{array}{c}
\dot{w}_{\mathrm{p}} \\
\ddot{v}_{\mathrm{p}}
\end{array}\right]\left[\begin{array}{c}
\dot{w}_{\mathrm{q}} \\
\ddot{v}_{\mathrm{q}}
\end{array}\right]^{T}\right]=\left[\begin{array}{ll}
Q & S \\
S^{\mathrm{T}} & R
\end{array}\right] \delta_{\mathrm{pq}}
$$

b) La séquence des entrées $u(k)$ est une séquence aléatoire quasi-stationnaire non-corrélée avec les bruits de process et de mesure [1]. Elle doit être complète en fréquences $[1,3]$.

c) Le système $(A, C)$ est observable. Ainsi pour un index donné $\beta$, on définit la « matrice d'observabilité étendue »

$$
\Gamma_{\beta}: \quad \Gamma_{\beta}=\left[\begin{array}{c}
C \\
C A \\
\vdots \\
C A^{\beta-1}
\end{array}\right]
$$

L'hypothèse c) implique que cette matrice est de rang plein dès que le nombre de lignes $\beta \times l$ est supérieur ou égal à l'ordre du système $2 n$.

\section{Estimation de l'erreur sur les paramètres}

Les méthodes d'identification des matrices dynamiques seront évoquées à la section 4. Rappelons simplement qu'elles fournissent les caractéristiques modales essentiellement à partir de l'identification de la matrice de transition $A$, et par là la matrice d'évolution continue $A_{\mathrm{c}}$.

L'estimation de l'erreur sur les paramètres modaux se fait par estimation de l'erreur sur les pôles complexes du système, c'est-à-dire les valeurs propres $\lambda_{k}$ de la matrice d'évolution $A_{\mathrm{c}}$. Ces valeurs propres sont elles-mêmes liées aux valeurs propres de la matrice $A$ :

$$
\mu_{k}=e^{\delta T \lambda_{k}}
$$
forme

On sait que la valeur propre peut se mettre sous la

$$
\lambda_{k}=-\omega_{k} \eta_{k}+j \omega_{k} \sqrt{1-\eta_{k}^{2}}
$$

où $\omega_{k}$ et $\eta_{k}$ sont respectivement la pulsation et le taux d'amortissement du $k^{\text {ième }}$ mode de la structure.

On définit l'erreur $\tilde{\mu}_{k}$ sur le pôle $\mu_{k}$ par la différence entre sa valeur identifiée $\hat{\mu}_{k}$ et sa valeur exacte $: \tilde{\mu}_{k}=$ $\hat{\mu}_{k}-\mu_{k}$.

Les covariances des erreurs $\tilde{\omega}_{k}$ sur les pulsations $\omega_{k}$ et $\tilde{\eta}_{k}$ sur les amortissements $\eta_{k}$ sont données par :

$$
\begin{aligned}
& E\left[\tilde{\omega}_{k}^{2}\right]=\frac{1}{4 \delta T^{2}}\left(\frac{E\left[\tilde{\mu}_{k}^{2}\right]}{\mu_{k}^{2}}-2 \frac{E\left[\tilde{\mu}_{k} \tilde{\mu}_{k}^{\mathrm{c}}\right]}{\mu_{k} \mu_{k}^{\mathrm{c}}}+\frac{E\left[\tilde{\mu}_{k}^{\mathrm{c} 2}\right]}{\mu_{k}^{\mathrm{c} 2}}\right) \\
& E\left[\tilde{\eta}_{k}^{2}\right]=\frac{1}{4 \delta T^{2} \hat{\omega}_{k}^{2}}\left(\frac{E\left[\tilde{\mu}_{k}^{2}\right]}{\mu_{k}^{2}}+\frac{2 E\left[\tilde{\mu}_{k} \tilde{\mu}_{k}^{\mathrm{c}}\right]}{\mu_{k} \mu_{k}^{\mathrm{c}}}+\frac{E\left[\tilde{\mu}_{k}^{c 2}\right]}{\mu_{k}^{c 2}}\right)
\end{aligned}
$$
lations.

On trouvera en annexe 1 la démonstration de ces re-

\section{Estimation de l'erreur sur les pôles complexes}

\subsection{Adaptation de la méthode des sous-espaces : N4SID}

Dans l'esprit de la méthode de sous-espaces N4SID, on définit, pour un index donné $\beta$ appelé « ordre du modèle $»$, la « matrice d'observabilité étendue $» \Gamma_{\beta}$ par l'équation (2.3), et suivant les procédures génériques $\left(\mathrm{N} 1\right.$ à N4), les matrices d'excitations $\Delta U_{\mathrm{p}}, \Delta U_{\mathrm{f}}$ du passé et du futur, de réponse (accélérations) $\ddot{Y}_{\mathrm{p}}, \ddot{Y}_{\mathrm{f}}$ du passé et du futur. 
L'élimination des bruits se fait par la méthode dite « de la variable instrumentale ». Dans le cas présent cette variable instrumentale est définie par la matrice

$$
\ddot{P}=\left[\begin{array}{l}
\Delta U_{\mathrm{p}} \\
\ddot{Y}_{\mathrm{p}}
\end{array}\right]
$$

par analogie avec celle couramment utilisée dans la littérature $[3,15,16]$. Pour en résumer grossièrement le principe, la méthode de la variable instrumentale consiste à corréler le signal de sortie avec un signal dit « variable instrumentale », qui lui soit fortement corrélé, de façon à conserver la dynamique du système, mais non-corrélé avec les bruits de process et de mesure. Cette opération a la particularité d'éliminer asymptotiquement les termes de bruit $[3,14]$. C'est le cas des signaux « du passé » qui figurent dans la définition (4.1), fortement corrélés avec les sorties «du futur », mais non-corrélés avec les bruits $\ll$ du futur $»$.

Pour réaliser concrètement la décorrélation, Van Overshee [3] utilise la projection oblique :

$$
\ddot{O}_{\beta}=\ddot{Y}_{\mathrm{f}} / \Delta U_{\mathrm{f}} \ddot{P}
$$

de l'espace ligne de la matrice $\ddot{Y}_{\mathrm{f}}$ sur l'espace ligne de la « variable instrumentale $» \ddot{P}$ parallèlement à l'espace ligne de la matrice $\Delta U_{\mathrm{f}}$. On démontre la propriété asymptotique :

$$
\ddot{O}_{\beta} \underset{j \rightarrow \infty}{=} \Gamma_{\beta} \dot{X}_{\mathrm{f}}
$$

où $\dot{X}_{\mathrm{f}}$ est une séquence (au sens de la procédure P2) d'estimés des vecteurs d'état du système.

On trouvera en annexe 2 la démonstration de ce résultat.

Il convient ensuite de réaliser une décomposition en valeurs singulières (SVD) de $\ddot{O}_{\beta}$. Après inspection des $\beta$ valeurs singulières de la matrice $S$, la décomposition est partitionnée comme suit :

$$
\ddot{O}_{\beta}=U S V^{\mathrm{T}}=\left[\begin{array}{ll}
U_{s} & U_{n}
\end{array}\right]\left[\begin{array}{ll}
S_{s} & 0 \\
0 & S_{n}
\end{array}\right]\left[\begin{array}{c}
V_{s}^{\mathrm{T}} \\
V_{n}^{\mathrm{T}}
\end{array}\right]
$$

où la matrice $S_{s}$ regroupe les $r$ valeurs singulières les plus significatives. La valeur $r$, dite « rang de coupure $»$ est choisie par l'utilisateur, en considérant que la matrice $S_{n}$ ne doit plus représenter que le sous-espace des bruits. Les estimations de $\Gamma_{\beta}$ et de $X_{\beta}$ sont alors obtenues à un changement de base arbitraire près.

$$
\hat{\Gamma}_{\beta}=U_{s} \quad \hat{\dot{X}}_{\mathrm{f}}=S_{s} V_{s}^{\mathrm{T}}
$$

Les estimations des matrices dynamiques $A$ et $C$ sont alors extraites en utilisant la propriété de « shift » de $\Gamma_{\beta}$. On définit deux matrices $\underline{\Gamma}_{\beta}$ et $\bar{\Gamma}_{\beta}$ par troncatures inférieures et supérieures de $\bar{\Gamma}_{\beta}$ :

$$
\underline{\Gamma}_{\beta}=\left[\begin{array}{c}
C \\
C A \\
\vdots \\
C A^{\beta-2}
\end{array}\right], \quad \bar{\Gamma}_{\beta}=\left[\begin{array}{c}
C A \\
C A^{2} \\
\vdots \\
C A^{\beta-1}
\end{array}\right]
$$

On a évidemment la propriété : $\bar{\Gamma}_{\beta}=\underline{\Gamma}_{\beta} A$. Une estimée $\hat{A}$ de la matrice $A$ est extraite à partir de la matrice estimée $\hat{\Gamma}_{\beta}$ par pseudo-inversion de cette relation soit : $\hat{A}=\underline{\Gamma}_{\beta}^{\dagger} \hat{\Gamma}_{\beta}$. Le premier bloc-ligne de $\hat{\Gamma}_{\beta}$ fournit une estimée $\hat{C}$ de la matrice $C$.

\subsection{Covariances des erreurs sur les pôles}

Il a été démontré $[7,17]$ que les erreurs $\tilde{\mu}_{k}$ sur les pôles de la matrice $A$ sont données par la relation :

$$
\tilde{\mu}_{k}=f_{k}^{*} \hat{U}_{s} U_{s}^{\mathrm{T}}\left(\Gamma_{\beta}^{\mathrm{d}}\right)_{\bullet k}
$$

où la matrice $\Gamma_{\beta}^{\mathrm{d}}$, matrice d'observabilité étendue en espace modal, est définie par : $\Gamma_{\beta}^{\mathrm{d}}=\Gamma_{\beta} \Phi^{-1}, \Phi$ étant la matrice des vecteurs propres de $A$, et le vecteur $f_{k}$ par :

$$
f_{k}^{*}=\left(J_{1} \Gamma_{\alpha}^{\mathrm{d}}\right)\left(J_{2}-J_{1} \mu_{k}\right)
$$

avec

$$
J_{1}=\left[\begin{array}{ll}
I_{(\alpha-1) l} & 0_{(\alpha-1) \times l}
\end{array}\right], J_{2}=\left[0_{(\alpha-1) \times l} I_{(\alpha-1) l}\right]
$$

Les covariances des erreurs sont alors obtenues en tenant compte des propriétés de la décomposition en valeurs singulières.

Ces covariances s'expriment comme suit :

$$
\begin{aligned}
\lim _{j \rightarrow \infty} E\left[\tilde{\mu}_{k}^{2}\right] & =\frac{1}{j} \sum_{|\tau|<\beta} \ddot{b}_{k}^{\mathrm{T}} R_{\ddot{\zeta} \ddot{\zeta}}(\tau) \ddot{b}_{k} f_{k}^{*} R_{\ddot{n}_{\mathrm{f}} \ddot{n}_{\mathrm{f}}}(\tau) f_{k}^{\mathrm{c}} \\
\lim _{j \rightarrow \infty} E\left[\tilde{\mu}_{k} \tilde{\mu}_{k}^{\mathrm{c}}\right] & =\frac{1}{j} \sum_{|\tau|<\beta} \ddot{b}_{k}^{\mathrm{T}} R_{\ddot{\zeta} \ddot{\zeta}}(\tau) \ddot{b}_{k}^{\mathrm{c}} f_{k}^{*} R_{\ddot{n}_{\mathrm{f}} \ddot{n}_{\mathrm{f}}}(\tau) f_{k} \\
\lim _{j \rightarrow \infty} E\left[\tilde{\mu}_{k}^{c^{2}}\right] & =\frac{1}{j} \sum_{|\tau|<\beta} \ddot{b}_{k}^{*} R_{\ddot{\zeta} \ddot{\zeta}}(\tau) \ddot{b}_{k}^{\mathrm{c}} f_{k}^{\mathrm{T}} R_{\ddot{n}_{\mathrm{f}} \ddot{n}_{\mathrm{f}}}(\tau) f_{k}
\end{aligned}
$$

où l'on a défini

$$
\ddot{b}_{h}=\lim _{j \rightarrow \infty} \ddot{H} \hat{U}_{s}^{\mathrm{T}}\left(\Gamma_{\beta}^{\mathrm{d}}\right)_{\bullet h}
$$

et où $R_{\ddot{n}_{\mathrm{f}} \ddot{n}_{\mathrm{f}}}$ est la matrice de covariance des bruits es-

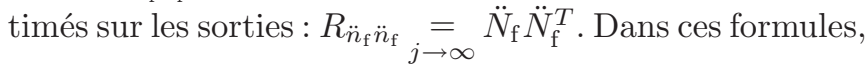
toutes les matrices sont définies asymptotiquement, et seront assimilées à leurs estimées.

On trouvera la démonstration de ces relations dans l'annexe 3 et le calcul de $N_{\mathrm{f}}$ en fin de l'annexe 2. Les mêmes développements démontrent par ailleurs la normalité asymptotique des erreurs $\tilde{\mu}_{k}$ sur les modes complexes.

Ces équations, avec les équations (3.3) et (3.4), permettent d'estimer les dispersions des paramètres modaux.

\section{Validation de la méthode en simulation}

La présente théorie a été testée en simulation sur un exemple de système masses-ressorts-amortisseurs. Dans un but de validation de la méthode, la distribution gaussienne des écarts d'identification prévue par la théorie sera comparée à celle observée à partir d'un grand nombre d'essais. 


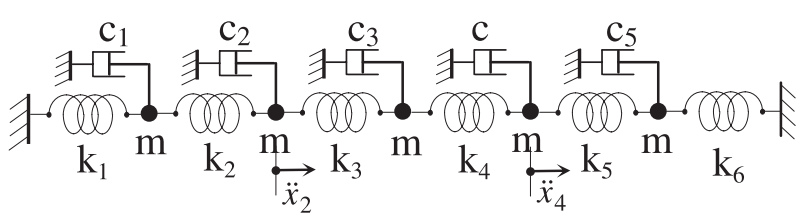

Fig. 1. Schéma du système simulé.

Tableau 1. Paramètres modaux du système simulé.

\begin{tabular}{lcr}
\hline Mode & Fréquence $(\mathrm{Hz})$ & Amortissement \\
\hline 1 & 19,28 & $3,1 \mathrm{E}-3$ \\
2 & 33,26 & $7,4 \mathrm{E}-4$ \\
3 & 52,13 & $1,6 \mathrm{E}-3$ \\
4 & 62,24 & $8,9 \mathrm{E}-4$ \\
5 & 78,84 & $6,4 \mathrm{E}-4$ \\
\hline
\end{tabular}

\subsection{Principe de validation}

En simulation les valeurs exactes des paramètres sont connues. La méthode précédente se trouvera validée si les erreurs d'estimation constatées pour un grand nombre d'essais suivent une loi gaussienne dont l'écart-type est identique à celui qui a été estimé par la théorie à l'aide d'un seul essai.

Dans un cas réel, les valeurs exactes des paramètres ne sont pas connues, mais peuvent être approchées assez précisément (dans une optique de validation) par une moyenne sur un grand nombre d'essais.

Pour une variable $x$ à identifier, de valeur exacte $\underline{x}$, l'écart-type estimé $s$ a lui-même une variabilité statistique. Pour mieux exploiter les résultats obtenus, la validation a été pratiquée ainsi : pour chaque essai $(i)$ l'erreur exacte d'identification, ici connue, est rapportée à l'écart type prévisionnel $s_{i}$ estimé à partir de cet essai. On définit ainsi, pour cet essai, le rapport :

$$
\rho_{i}=\frac{x_{i}-\underline{x}}{s_{i}} ; i=1 \ldots j
$$

$x_{i}$ étant la valeur identifiée. La théorie précédente se trouvera validée si ce rapport suit une loi gaussienne centrée réduite. En pratique, on se contentera de vérifier les valeurs des bornes de confiance à $95 \%$, soit \pm 2 , et à $50 \%$, soit $\pm 0,7$.

\subsection{Système simulé}

Le système simulé, dont le schéma est présenté figure 1 , possède cinq degrés de liberté.

En utilisant les équations d'état (2.2), le présent système est simulé sous les conditions suivantes. Deux forces d'excitation aléatoires sont appliquées sur les masses 2 et 4 . En ces mêmes points, deux signaux d'accélérations sont collectés, sur lesquels des signaux correspondant à des bruits blancs sont ajoutés. Ce bruit correspond à un rapport signal sur bruit d'environ 0,5\%.

250 fichiers de 4000 points ont été analysés pour obtenir les diagrammes de distributions présentés. Seul

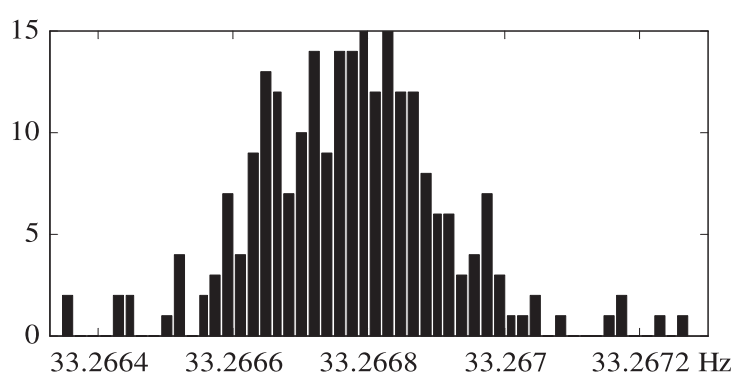

Fig. 2. Deuxième mode - Distribution de la fréquence.

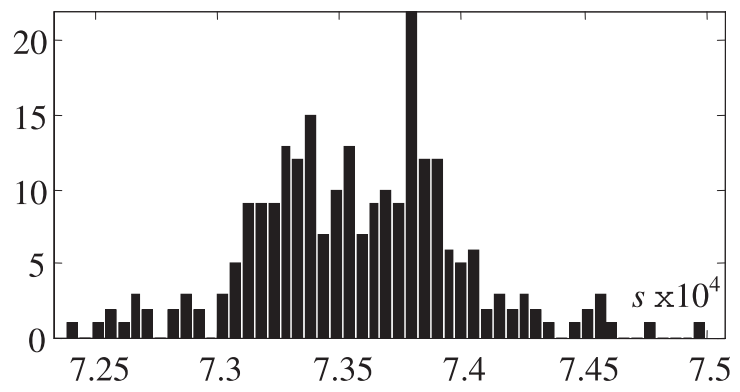

Fig. 3. Deuxième mode - Distribution du taux d'amortissement.

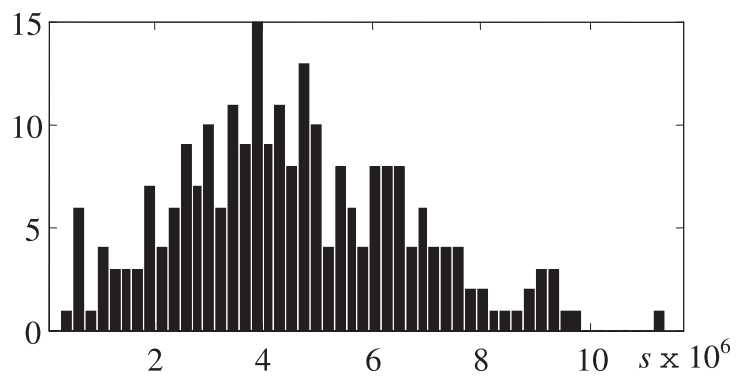

Fig. 4. Deuxième mode - Distribution de l'écart-type estimé pour le taux d'amortissement.

le second mode jugé particulièrement représentatif sera présenté en détail.

Les figures 2 et 3 montrent les distributions de la fréquence et du taux d'amortissement du second mode, obtenus après identification en utilisant un modèle d'ordre $\beta$ égal à 12 et un rang de coupure $r$ égal à 10 . Les distributions ont une allure gaussienne centrée autour des valeurs théoriques de ces paramètres

\subsection{Estimation de la dispersion}

La figure 4 montre la distribution des écart-types estimés par la théorie pour le taux d'amortissement du deuxième mode.

Les figures 5 et 6 montrent les distributions du ratio $\rho$ (cf. Sect. 5.1) pour la fréquence et pour le taux d'amortissement. Sur ces diagrammes, on constate que les critères de normalité gaussienne à $95 \%$ (intervalle $[-2-+2]$ ) et à $50 \%$ (intervalle $[-0,7-+0,7])$ sont assez bien respectés. Ce résultat confirme la distribution gaussienne centrée réduite prévue par la théorie. Des résultats similaires ont été obtenus pour les autres modes. 


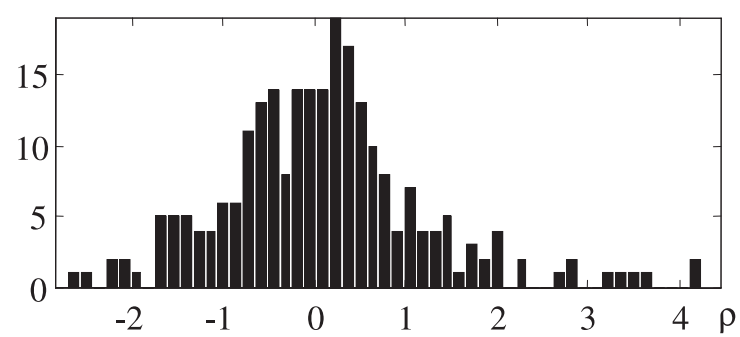

Fig. 5. Deuxième mode - Distribution du ratio $\rho$ pour la fréquence.

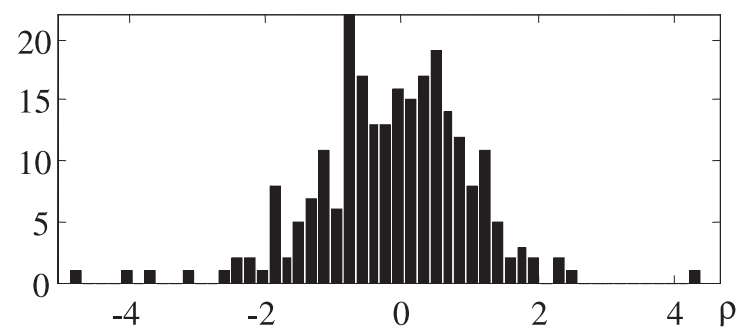

Fig. 6. Deuxième mode - Distribution du ratio $\rho$ pour le taux d'amortissement.

\section{Application et validation sur un cas expérimental}

\subsection{Schéma du dispositif}

La structure expérimentale, schématisée sur la figure 7 , représente grossièrement l'ossature d'un immeuble de deux étages. Cette structure possède six modes majeurs. Cependant, étant excitée comme le montre la figure, quatre de ceux-ci, appelés modes « principaux », seront fortement excités, et deux, appelés modes « secondaires », le seront plus faiblement. Les modes « principaux» sont la translation des planchers en phase et la translation des planchers en opposition, dans la direction d'excitation, la rotation des planchers en phase et la rotation des planchers en opposition. Les modes « secondaires» sont la translation des planchers en phase et la translation des planchers en opposition, dans la direction perpendiculaire à l'excitation. Il existe aussi un certain nombre de modes locaux dans la structure comme les modes de vibrations des plaques des planchers, les modes de barres, mais ceuxci sont très faiblement excités.

La structure est excitée aléatoirement à l'aide d'un pot vibrant près de l'un de ses sommets, les réponses étant recueillies en deux points par des accéléromètres. 259 fichiers de 4096 points sont analysés à l'aide du processus d'identification de sous-espaces, pour un ordre de modèle $\beta$ égal à 40 et un rang de coupure $r$ égal à 30 . Les fonctions de transfert sont présentées sur la figure 8. Les paramètres modaux des modes significatifs sont présentés, à titre indicatif, dans le tableau 2. On observera que les amortissements sont très faibles, comme il est d'usage pour des structures métalliques entièrement soudées. Par ailleurs, on sait que dans ce type de configuration, les fréquences sont obtenues avec une bonne précision, tandis que les

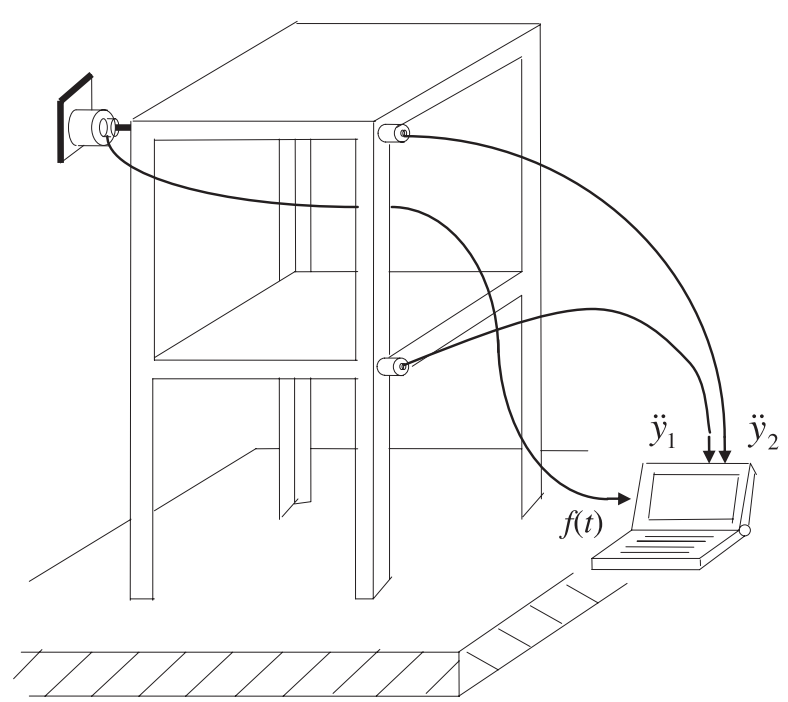

Fig. 7. Structure expérimentale.

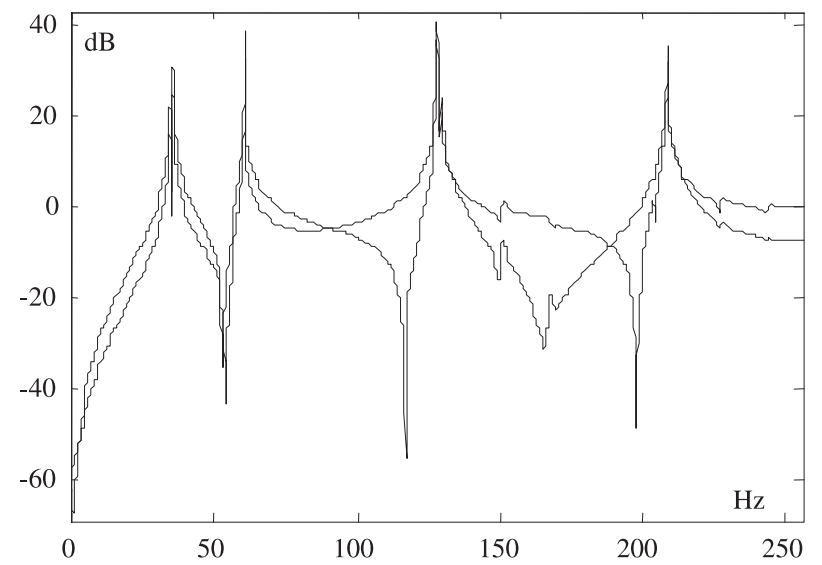

Fig. 8. Fonctions de transfert expérimentales de la structure : sorties 1 et 2 .

Tableau 2. Paramètres modaux expérimentaux, ${ }^{*}$ : Modes principaux, ${ }^{* *}$ : modes secondaires.

\begin{tabular}{lcccc}
\hline Mode & $\begin{array}{c}\text { Fréquence } \\
(\mathrm{Hz})\end{array}$ & Amortissement & $\begin{array}{c}\text { Sortie 1 } \\
(\mathrm{dB})\end{array}$ & $\begin{array}{c}\text { Sortie 2 } \\
(\mathrm{dB})\end{array}$ \\
\hline $1^{* *}$ & 34,43 & $4,18 \mathrm{E}-3$ & 22,27 & 15,84 \\
$2^{*}$ & 36,22 & $3,84 \mathrm{E}-3$ & 30,77 & 24,91 \\
$3^{*}$ & 60,88 & $1,026 \mathrm{E}-3$ & 38,92 & 33,13 \\
$4^{*}$ & 128,34 & $7,85 \mathrm{E}-4$ & 36,95 & 40,64 \\
$5^{* *}$ & 129,37 & $7,74 \mathrm{E}-4$ & 19,55 & 23,59 \\
$6^{*}$ & 208,62 & $9,08 \mathrm{E}-4$ & 32,52 & 35,12 \\
\hline
\end{tabular}

taux d'amortissement sont obtenus avec une assez mauvaise précision.

\subsection{Estimation de l'erreur}

L'analyse des résultats a mis en évidence une légère évolution continue des paramètres modaux entre le premier et le dernier enregistrement effectués. Les évolutions de la fréquence et du taux d'amortissement du deuxième 


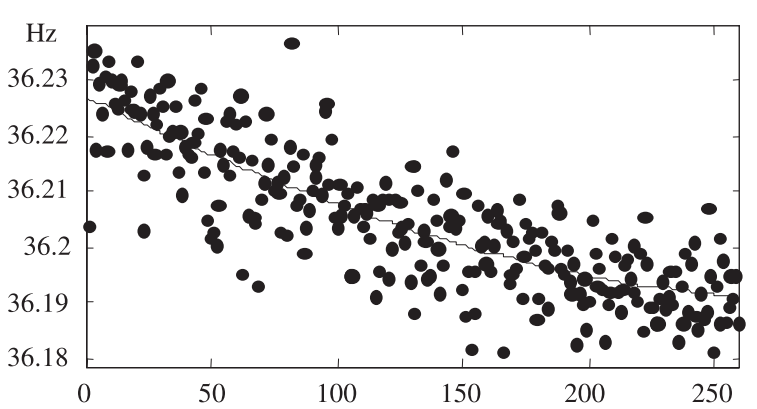

Fig. 9. Deuxième mode - Évolution de la fréquence en fonction du numéro de fichier analysé.

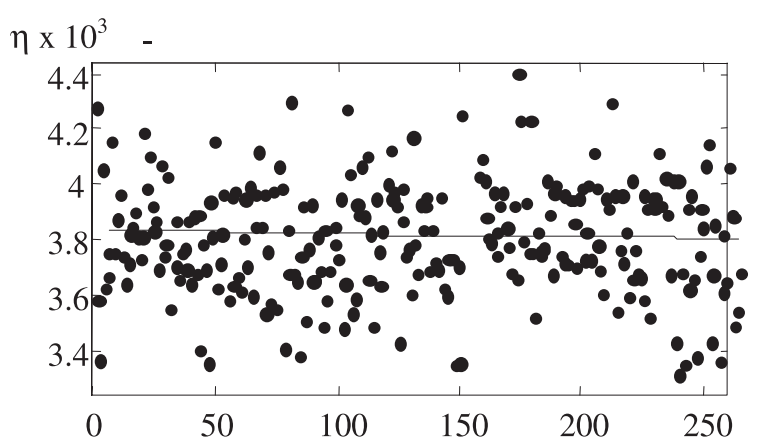

Fig. 10. Deuxième mode - Évolution du taux d'amortissement en fonction du numéro de fichier analysé.

mode sont présentées sur les figures 9 et 10. Chaque valeur obtenue est représentée par un point $(\bullet)$. Cette évolution, qui doit probablement être attribuée à une évolution de la raideur de scellement des pieds de structure sur le sol en béton, n'a pu être maîtrisée. Bien que faible numériquement, elle complique quelque peu la validation envisagée, car elle est d'un ordre de grandeur comparable aux erreurs faisant l'objet de l'analyse.

Pour pallier à cette difficulté, il a été défini, pour chaque paramètre modal, une courbe moyenne à l'aide d'un outil standard de lissage polynomial. Ces courbes figurent sur les figures 9 et 10 . Ainsi pour une variable $x$, à l'essai $(i)$ peuvent maintenant être associées :

- la valeur $x_{i}$ de la variable identifiée

- la valeur $\underline{x}_{i}$ de la courbe moyenne, considérée comme valeur de référence (quasi-exacte).

- une dispersion estimée $s_{i}$

La valeur $\rho_{i}$ définie à la section 4.2 est remplacée par le ratio suivant :

$$
\rho_{i}=\frac{x_{i}-\underline{x}_{i}}{s_{i}} ; i=1 \ldots j
$$

Les critères de normalité à $95 \%$ et à $50 \%$ sont appliqués comme définis à la section 4.1

Les figures 11 et 12 présentent respectivement les distributions des rapports $\rho$ pour la fréquence et pour le taux d'amortissement, pour le second mode. Pour ces deux distributions, les critères de distribution gaussienne à $95 \%$ et à $50 \%$ sont assez bien respectés. Ce résultat confirme la distribution gaussienne centrée réduite prévue par la théorie.

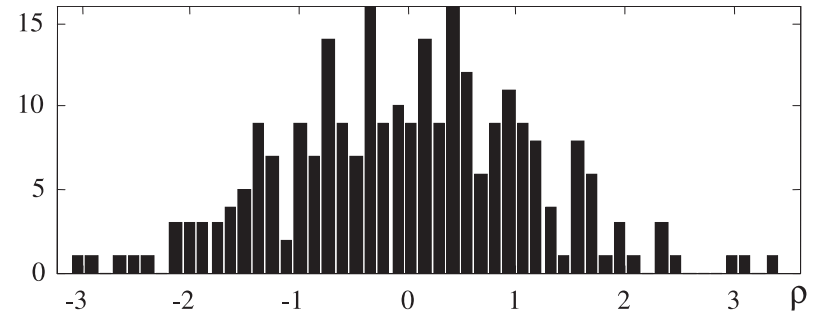

Fig. 11. Second mode - distribution du rapport $\rho$ pour la fréquence.

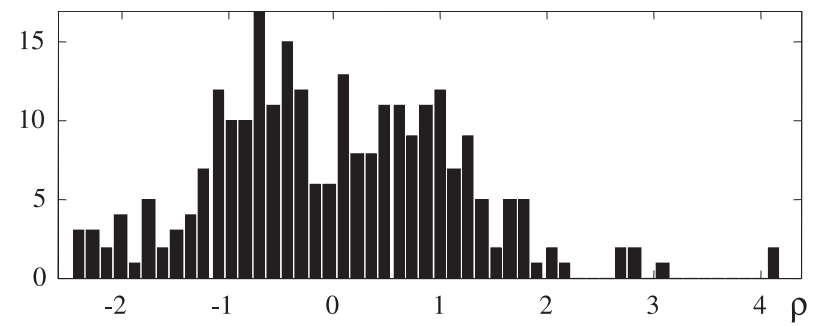

Fig. 12. Second mode - distribution du rapport $\rho$ pour le taux d'amortissement.

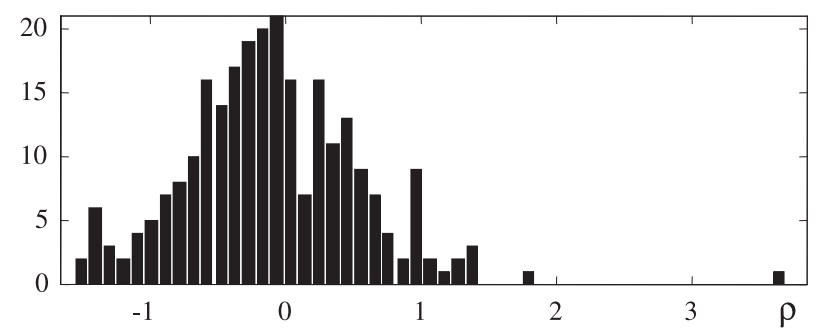

Fig. 13. Cinquième mode - distribution du rapport $\rho$ pour la fréquence.

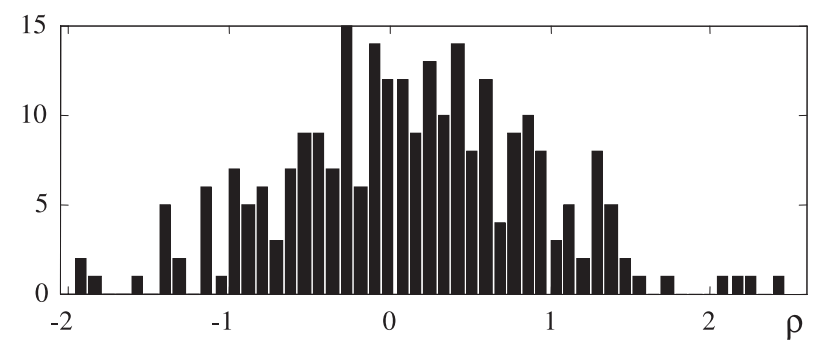

Fig. 14. Cinquième mode - distribution du rapport $\rho$ pour le taux d'amortissement.

Les autres modes conduisent à des résultats du même type, cependant quelquefois avec une surestimation de la variance estimée. Par exemple, pour le cinquième mode, les figures 13 et 14 montrent des distributions du rapport $\rho$ plus étroites, ce qui correspond à une surestimation de l'erreur statistique sur les paramètres de ce mode.

\section{Conclusions}

L'étude précédente a permis d'établir une formulation théorique de la variance des paramètres modaux, fréquence et amortissement, fournis par une analyse 
d'identification, dans le cadre déterministe où l'excitation est mesurée. Ce résultat doit permettre en principe, à partir d'une opération d'identification unique, de proposer pour chaque paramètre modal identifié une fourchette prévisionnelle de validité et par là une appréciation chiffrée de la valeur des résultats annoncés.

La théorie a d'abord été validée à travers un exemple de pure simulation, pour un système à 5 degrés de liberté en présence de bruits de mesure et de process. Une analyse statistique basée sur un grand nombre de simulations a permis de confirmer les formules des covariances prévues par la théorie, ainsi que les propriétés statistiques des erreurs d'identification.

La méthode a ensuite été appliquée à un cas expérimental. La validation de la méthode est faite à nouveau à partir d'une importante série d'essais expérimentaux. La dispersion prévue par la théorie à partir d'un essai unique est confirmée par l'analyse statistique, pour certains modes, quelquefois surestimée pour d'autres modes. Les résultats montrent donc qu'à partir d'une opération d'identification unique, il est possible de fournir une «fourchette» de validité correcte, éventuellement surestimée, ce qui va dans un sens favorable pour le praticien.

La surestimation rencontrée pour certains modes peut être attribuée au contexte expérimental. Dans le cas présent l'évolution observée des paramètres modaux en cours d'analyse, l'existence probable de petites nonlinéarités, la présence de bruits de mesure et de process probablement colorés, qui provoquent des perturbations d'un ordre de grandeur comparable à celui des erreurs analysées, sont des causes probables d'imprécision sur le résultat. En particulier ces perturbations impliquent un choix difficile au niveau de l'ordre estimé du modèle $\beta$ et du rang de coupure $r$. Or ce choix conditionne fortement la qualité de l'identification, et donc par là la valeur de l'erreur statistique fournie. Il est à noter qu'à ce jour n'existe aucune méthode confirmée permettant une détermination optimale de ces paramètres.

Malgré ces restrictions il ressort de la présente étude qu'une estimation fiable, ou tout au moins « conservative » de la fourchette d'erreur sur un paramètre identifié est possible dans les cas pratiques, et cela à l'issue d'une opération unique d'identification.

\section{Annexes}

\section{Annexe 1. Erreur sur les paramètres modaux}

Elle se déduit de l'erreur sur valeurs propres $\lambda_{k}$ de la matrice d'évolution $A_{\mathrm{c}}$ données par l'équation (3.2).

Soit $\tilde{\lambda}_{k}$ l'erreur sur la valeur de $\lambda_{k}$. Considérant que les taux d'amortissement sont petits, et que l'estimation des pulsations modales $\omega_{k}$ est proche au second ordre de leur valeur réelle, on obtient :

$$
\tilde{\lambda}_{k}+\tilde{\lambda}_{k}^{c} \approx-2 \hat{\omega}_{k} \tilde{\eta}_{k} \quad \text { et } \quad \tilde{\lambda}_{k}-\tilde{\lambda}_{k}^{c} \approx 2 i \tilde{\omega}_{k}
$$

En posant $\tilde{\rho}_{k}=-\frac{1}{2}\left(\tilde{\lambda}_{k}+\tilde{\lambda}_{k}^{c}\right)$ il vient

$$
E\left[\tilde{\rho}_{k}^{2}\right]=E\left[\left(\hat{\omega}_{k} \tilde{\eta}_{k}\right)^{2}\right] \approx \hat{\omega}_{k}^{2} E\left[\tilde{\eta}_{k}^{2}\right]
$$

et

$$
E\left[\tilde{\omega}_{k}^{2}\right] \approx-\frac{1}{4} E\left[\tilde{\lambda}_{k}^{2}-2 \tilde{\lambda}_{k} \tilde{\lambda}_{k}^{c}+\tilde{\lambda}_{k}^{c^{2}}\right]
$$

Par différenciation de l'équation (3.1), on obtient :

$$
\tilde{\lambda}_{k}=\frac{1}{\delta T} \frac{\tilde{\mu}_{k}}{\mu_{k}}
$$

Les équations (3.3) et (3.4) s'en déduisent alors directement.

\section{Annexe 2. Factorisation de la matrice « observation »}

À partir du système d'équations (2.2) compte tenu de la procédure (N1), on prouve facilement :

$$
\begin{aligned}
\ddot{y}_{k} & =\Gamma_{i} \dot{x}_{k}+H_{i} \delta u_{k}+\Psi_{i} \dot{w}_{k}+\ddot{v}_{k} \\
\dot{x}(k+i) & =A^{i} \dot{x}(k)+\Delta_{i} \delta u(k)+E_{i} \dot{w}(k)
\end{aligned}
$$

où $\Gamma_{i}$ et $\Delta_{i}$ sont appelées matrices « d'observabilité » et de « contrôlabilité » étendues. $H_{i}$ est attachée au vecteur d'excitation, et les deux matrices $\Psi_{i}$ et $\mathrm{E}_{i}$ aux effets aléatoires. Les matrices ont les structures suivantes :

$$
\begin{aligned}
H_{i} & =\left[\begin{array}{cccc}
D & 0 & \cdots & 0 \\
C B & D & \ddots & \vdots \\
\vdots & \ddots & D & 0 \\
C A^{i-2} B & \cdots & C B & D
\end{array}\right] \\
\Psi_{i} & =\left[\begin{array}{cccc}
0 & 0 & \cdots & 0 \\
C & 0 & \ddots & \vdots \\
\vdots & C & 0 & 0 \\
C A^{i-2} & \cdots & C & 0
\end{array}\right], \\
\Delta_{i} & =\left[\begin{array}{llll}
A^{i-1} B & \cdots & A B & B
\end{array}\right], \quad E_{i}=\left[\begin{array}{lll}
A^{i-1} & \cdots & A I
\end{array}\right]
\end{aligned}
$$

En utilisant les procédures génériques (N1 à N4) et les procédures spécifiques (P2 à P3), on établit les relations suivantes :

$$
\left\{\begin{array}{l}
\ddot{Y}_{p}=\Gamma_{\alpha} \dot{X}_{p}+H_{\alpha} \Delta U_{p}+\Psi_{\alpha} \dot{W}_{p}+\ddot{V}_{p} \\
\ddot{Y}_{\mathrm{f}}=\Gamma_{\beta} \dot{X}_{\mathrm{f}}+H_{\beta} \Delta U_{\mathrm{f}}+\Psi_{\beta} \dot{W}_{\mathrm{f}}+\ddot{V}_{\mathrm{f}} \\
\dot{X}_{\mathrm{f}}=A^{\beta} \dot{X}_{p}+\Delta_{\beta} \Delta U_{p}+\mathrm{E}_{\beta} \dot{W}_{p}
\end{array}\right.
$$

La «variable instrumentale » retenue est définie par l'équation (4.1).

On démontre $[3,14]$ que la projection oblique $\ddot{O}_{\beta}=$ $\ddot{Y}_{\mathrm{f}} / \Delta U_{\mathrm{f}} \ddot{P}$ vérifie $\ddot{O}_{\beta}=\hat{\Gamma}_{\beta} \hat{\dot{X}}_{\mathrm{f}}+\ddot{N}_{\mathrm{f}}$ où le terme de bruit $\ddot{N}_{\mathrm{f}}=\Psi_{\beta} \dot{W}_{\mathrm{f}}+\ddot{V}_{\mathrm{f}}$ tend vers zéro lorsque la longueur $j$ de la séquence tend vers l'infini, ce qui démontre la relation (4.3).

Notons que l'équation (A2.1 b) définit le terme de bruit $\ddot{N}_{\mathrm{f}}$, soit : $\ddot{N}_{\mathrm{f}}=\ddot{Y}_{\mathrm{f}}-\ddot{O}_{\beta}-H_{\beta} \Delta U_{\mathrm{f}}$. 


\section{Annexe 3. Covariance des erreurs sur les paramètres}

À partir de la matrice « observation $» \ddot{O}_{\beta}$ définie par l'équation (4.2), la SVD de $\ddot{O}_{\beta}$ peut s'écrire

$$
\ddot{O}_{\beta}=\hat{U}_{s} \hat{S}_{s} \hat{V}_{s}^{\mathrm{T}}+\hat{U}_{n} \hat{S}_{n} \hat{V}_{n}^{\mathrm{T}}
$$

Multipliant les deux membres par $\hat{V}_{s}$ à droite, compte tenu de la définition de $\ddot{O}_{\beta}$ et de la formule de projection oblique on obtient : $\ddot{Y}_{\mathrm{f}} \prod_{\Delta U_{\mathrm{f}}^{\perp}} \ddot{P}^{\mathrm{T}} \hat{W}_{\mathrm{c}} \hat{V}_{s}=\hat{U}_{s} \hat{S}_{s}$ où l'on a posé :

$$
\hat{W}_{\mathrm{c}}=\left(\ddot{P} \prod_{\Delta U_{\mathrm{f}}^{\perp}} \ddot{P}^{\mathrm{T}}\right)^{-1} \ddot{P}
$$

Soient deux vecteurs déterministes $\xi$ et $\varsigma$ satisfaisant à la relation

$$
\xi^{*} \Gamma_{\beta}=\varsigma^{*} \Gamma_{\beta}=0
$$

En multipliant par $\xi^{*}$ à gauche et $\operatorname{par} \hat{S}_{s}^{-1}$ à droite l'équation (A3.1) devient

$$
\xi^{*} \ddot{N}_{\mathrm{f}} \prod_{\Delta U_{\mathrm{f}}^{\perp}} \ddot{P}^{\mathrm{T}} \hat{W}_{\mathrm{c}} \hat{V}_{s} \hat{S}_{s}^{-1}=\xi^{*} \hat{U}_{s}
$$

En utilisant la définition de l'opérateur de projection orthogonale complémentaire $\prod_{\Delta U_{\mathrm{f}}^{\perp}}$, il vient :

$$
\begin{aligned}
\frac{1}{\sqrt{j}} \xi^{*} \hat{U}_{s}=\xi^{*}\left(\left(\frac{1}{\sqrt{j}} \ddot{N}_{\mathrm{f}} \ddot{P}^{\mathrm{T}}\right)\right. & \\
& \left.-\left(\frac{1}{\sqrt{j}} \ddot{N}_{\mathrm{f}} \Delta U_{\mathrm{f}}^{\mathrm{T}}\right) R_{\mathrm{ff}}^{-1} R_{\mathrm{fp}}\right) \hat{W}_{\mathrm{c}} \hat{V}_{s} S_{s}^{-1}
\end{aligned}
$$

avec $R_{\mathrm{ff}}=\Delta U_{\mathrm{f}} \Delta U_{\mathrm{f}}^{\mathrm{T}}$ et $R_{\mathrm{fp}}=\Delta U_{\mathrm{f}} \ddot{P}^{\mathrm{T}}$

À partir des hypothèses a) et b) du paragraphe 1 , le Théorème Central Limite montre que les éléments suivants : $\frac{1}{\sqrt{j}} \ddot{N}_{\mathrm{f}} \ddot{P}^{\mathrm{T}}$ et $\frac{1}{\sqrt{j}} \ddot{N}_{\mathrm{f}} \Delta U_{\mathrm{f}}^{\mathrm{T}}$ ont des distributions asymptotiquement gaussiennes de moyenne nulle. En conséquence, la distribution asymptotique de $\sqrt{j} \xi^{*} \hat{U}_{s}$ peut s' écrire :

$$
\sqrt{j} \xi^{*} \hat{U}_{s} \underset{j \rightarrow \infty}{=} \xi^{*}\left(\frac{1}{\sqrt{j}} \ddot{N}_{\mathrm{f}} \ddot{Z}^{\mathrm{T}}\right) \ddot{H}
$$

avec

$$
\ddot{Z}=\left[\begin{array}{l}
\Delta U_{\mathrm{f}} \\
\Delta U_{\mathrm{p}} \\
\ddot{Y}_{\mathrm{p}}
\end{array}\right]=\left[\begin{array}{llll}
\ddot{\zeta}_{k} & \ddot{\zeta}_{k+1} & \cdots & \ddot{\zeta}_{k+j-1}
\end{array}\right]
$$

et

$$
\ddot{H}=j\left[\begin{array}{l}
-R_{\mathrm{ff}}^{-1} R_{\mathrm{fp}} \\
I
\end{array}\right] \hat{W}_{\mathrm{c}} \hat{V}_{s} \hat{S}_{s}^{-1}
$$

En remplaçant $\xi^{*}$ par $f_{k}^{*}$, et $\varsigma^{*}$ par $f_{l}^{*}$ dans cette équation, puis en multipliant les deux membres par $\hat{U}_{s}^{\mathrm{T}}\left(\Gamma_{\beta}^{\mathrm{d}}\right)_{\bullet k}$, on obtient les relations suivantes, valables asymptotiquement :

$$
\begin{aligned}
& \sqrt{j} f_{k}^{*} \hat{U}_{s} \hat{U}_{s}^{\mathrm{T}}\left(\Gamma_{\beta}^{\mathrm{d}}\right)_{\bullet k}=f_{k}^{*}\left(\frac{1}{\sqrt{j}} \ddot{N}_{\mathrm{f}} \ddot{Z}^{\mathrm{T}}\right) \ddot{b}_{k} \\
& \sqrt{j} f_{l}^{*} \hat{U}_{s} \hat{U}_{s}^{\mathrm{T}}\left(\Gamma_{\beta}^{\mathrm{d}}\right)_{\bullet l}=f_{l}^{*}\left(\frac{1}{\sqrt{j}} \ddot{N}_{\mathrm{f}} \ddot{Z}^{\mathrm{T}}\right) \ddot{b}_{l}
\end{aligned}
$$

où $\ddot{b}_{h}$ est défini par l'équation (4.6).

Il est facile de prouver [7] que $f_{k}^{*}$ est orthogonal à $\Gamma_{\beta}$.

Viberg et al. $[5,6,16]$ ont montré que $f_{k}^{*} \hat{U}_{s}$ a asymptotiquement une distribution gaussienne de moyenne nulle, et dont les covariances des éléments s'écrivent :

$$
\begin{gathered}
\lim _{j \rightarrow \infty} E\left[\left(f_{k}^{*} \hat{U}_{s}\right)^{\mathrm{T}}\left(f_{l}^{*} \hat{U}_{s}\right)\right]= \\
\frac{1}{j} \sum_{|\tau|<\beta} \ddot{H}^{\mathrm{T}} R_{\ddot{\zeta} \ddot{\zeta}}(\tau) \ddot{H} f_{l}^{*} R_{\ddot{n}_{\mathrm{f}} \ddot{n}_{\mathrm{f}}}(\tau) f_{l}^{\mathrm{c}} \\
\lim _{j \rightarrow \infty} E\left[\left(f_{k}^{*} \hat{U}_{s}\right)^{\mathrm{T}}\left(f_{l}^{*} \hat{U}_{s}\right)^{\mathrm{c}}\right]= \\
\frac{1}{j} \sum_{|\tau|<\beta} \ddot{H}^{\mathrm{T}} R_{\ddot{\zeta} \ddot{\zeta}}(\tau) \ddot{H}^{\mathrm{c}} f_{l}^{*} R_{\ddot{n}_{\mathrm{f}} \ddot{n}_{\mathrm{f}}}(\tau) f_{l} \\
\lim _{j \rightarrow \infty} E\left[\left(f_{k}^{*} \hat{U}_{s}\right)^{*}\left(f_{l}^{*} \hat{U}_{s}\right)^{\mathrm{c}}\right]= \\
\frac{1}{j} \sum_{|\tau|<\beta} \ddot{H}^{*} R_{\ddot{\zeta} \ddot{\zeta}}(\tau) \ddot{H}^{\mathrm{c}} f_{l}^{\mathrm{T}} R_{\ddot{n}_{\mathrm{f}} \ddot{n}_{\mathrm{f}}}(\tau) f_{l}
\end{gathered}
$$

La normalité asymptotique de $f_{k}^{*} \hat{U}_{s}$ implique celle des erreurs $\tilde{\mu}_{k}$ données par l'équation (4.4). Les formules ci-dessus peuvent être appliquées directement à ces erreurs, et conduisent aux équations $(4.5 \mathrm{a}-\mathrm{c})$. Enfin ces covariances peuvent être prises en compte directement dans les équations (3.3) et (3.4), qui expriment les covariances des erreurs sur les paramètres modaux.

\section{Références}

[1] L. Ljung, System Identification : Theory for the User, Prentice-Hall, Englewood Cliffs, NJ, 1987

[2] D. Ewins, Modal Testing : Theory and Practice, Research Studies Press Ltd, Somerset, England, 1995

[3] P. Van Overschee, Subspace Identification : Theory Implementation - Application, Ph.d. Thesis, Katholieke Universiteit Leuven, Belgium, February 1995

[4] M. Viberg, Subspace-based methods for the identification of linear time-invariant systems, Automatica 31 (1995) $1835-1851$

[5] M. Viberg, B. Ottersten, B. Wahlberg, L. Ljung, A statistical perspective on state-space modeling using subspace methods, Proceedings 30th IEEE Conference on Decision and Control, Brighton, England, 1991, pp. 1337-1342

[6] M. Viberg, B. Wahlberg, B. Ottersten, Analysis of state space system identification methods based on instrumental variables and subspace fitting, Automatica 33 (1997) 1603-1616

[7] M. Jansson, B. Wahlberg, A linear regression approach to state-space subspace system identification, Signal Processing 52 (1996) 103-129

[8] D. Bauer, M.Deistler, W. Scherrer, The analysis of the asymptotic variance of subspace algorithms, IFAC, System Identification, Fukuoka, Japan, 1997, pp. 1037-1041 
[9] D. Bauer, M. Deistler, W. Scherrer, Consistency and asymptotic normality of some subspace algorithms for systems without observed inputs, Automatica 35 (1999) 1243-1254

[10] D. Bauer, M. Jansson, Analysis of the asymptotic properties of the MOESP type of subspace algorithms, Automatica 36 (2000) 497-509

[11] M. Deistler, K. Peternell, W. Scherrer, Consistency and relative efficiency of subspace methods, Automatica 31 (1995) 1865-1875

[12] K. Peternell, W. Scherrer, M. Deistler, Statistical analysis of novel subspace identification methods, Signal Processing 52 (1996) 161-177

[13] T. Knudsen, Consistency analysis of subspace identification methods based on a linear regression approach, Automatica 37 (2001) 81-89
[14] M. Raffy, C. Gontier, A Subspace method of modal analysis using acceleration signals, Proceedings 20th IMAC, Los Angeles, USA, 2002, pp. 824-830

[15] B. Ottersten, M. Viberg, A subspace-based instrumental variable method for state-space system identification, 10th IFAC Symposium on system identification, Copenhagen, Denmark, 1994

[16] M. Viberg, B. Ottersten, B. Wahlberg, L. Ljung, Performance of subspace-based system identification methods, Proceedings IFAC 93, 7, Sydney, Australia, 1993, 369-372

[17] M. Raffy, C. Gontier, Statistical error on damping ratios in deterministic subspace identification, Proceedings 21th IMAC, Kissimmee, Florida, USA, 2003 